\title{
Measurement of the inclusive production cross sections for forward jets and forward - central dijets in CMS at $\sqrt{s}=7 \mathrm{TeV}$
}

\author{
Salim Cerci*t \\ Adiyaman University \\ E-mail: Salim.Cerciecern.ch
}

\begin{abstract}
We present the measurements of inclusive production cross sections for forward jets, as well for jets in dijet events with at least one jet emitted at central and the other at forward pseudorapidities in proton-proton collisions at $\sqrt{s}=7 \mathrm{TeV}$. The measurements were performed in the range of transverse momenta $p_{T}=35-150 \mathrm{GeV} / \mathrm{c}$ forward jets within pseudorapidities $3.2<|\eta|<4.7$, and central jets within the $|\eta|<2.8$ range. The differential cross sections $d^{2} \sigma / d p_{T} d \eta$ are compared to predictions from three approaches in perturbative quantum chromodynamics: (i) nextto-leading-order calculations obtained with and without matching to parton-shower Monte Carlo simulations, (ii) PYTHIA and HERWIG parton-shower event generators with different tunes of parameters, and (iii) CASCADE and HEJ models, including different non-collinear corrections to standard single-parton radiation.
\end{abstract}

36th International Conference on High Energy Physics,

July 4-11, 2012

Melbourne, Australia

\footnotetext{
*Speaker.

${ }^{\dagger}$ On behalf of the CMS Collaboration
} 


\section{Introduction}

Jet production in hadron-hadron collisions is sensitive to the underlying partonic QCD processes, to the details of parton radiation and to the parton density functions (PDFs) of the colliding hadrons. At previous colliders, the measured jet cross sections at large transverse momenta $\left(p_{T}\right)$ are successfully described by perturbative quantum chromodynamics (pQCD) calculations over several orders of magnitude [1]. The measurements, however, are limited to central pseudo-rapidities $|\eta|<2.4$, where the momentum fractions $x_{1}, x_{2}$ of the incoming partons are of the same order. Jets produced in the forward (or backward) hemispheres result from scatterings between colliding partons with increasingly different momentum fractions $x_{2} \ll x_{1}$, and thus allow one to study QCD in the small- $x$ region where PDFs are less well constrained by DIS data, and where deviations of the parton dynamics beyond the standard Dokshitzer-Gribov-Lipatov-Altarelli-Parisi (DGLAP) evolution equations [2] e.g. of the Balitski-Fadin-Kuraev-Lipatov (BFKL) [3], Ciafaloni-CataniFiorani-Marchesini (CCFM) [4] or gluon saturation [5] are expected. In the phase space considered in this paper, gluons participate in about $80 \%$ of the partonic interactions that lead to forward jet production, with paired parton momentum fractions of the order of $x_{2} \approx 10^{-4}$ and $x_{1} \approx 0.2$ [6]. Additionally, beyond the QCD motivation, jet measurements in the forward region are of interest for vector-boson-fusion processes where a Higgs boson can be produced in conjunction with forward and backward jets with $p_{T} \approx 40 \mathrm{GeV} / \mathrm{c}$.

\section{Experimental aspect}

A detailed description of the CMS detector can be found in Ref. [7], and the features most relevant to the present analysis are described below. The relevant detector components for the reconstruction of jets in this work are the ECAL and HCAL central calorimeters [8], as well as the HF forward calorimeters [9]. The ECAL cells are grouped in quasi-projective towers of granularity in pseudorapidity and azimuthal angle of $\Delta \eta \times \Delta \phi=0.0174 \times 0.0174$ in the barrel $(|\eta|<1.5)$, and of $0.05 \times 0.05$ in the endcap $(1.5<|\eta|<3.0)$. The HCAL has a tower granularity as small as $\Delta \eta \times \Delta \phi=0.087 \times 0.087$. The HCAL, when combined with the ECAL, measures jets with a resolution $\Delta E / E \approx 100 \% / \sqrt{E} \oplus 5 \%$. The HF calorimeters consist of steel absorbers containing embedded radiation-hard quartz fibres, located at $\pm 11.2 \mathrm{~m}$ from the centre of the CMS detector, and cover the region $2.9<|\eta|<5$.2. Half of the fibres run over the full longitudinal depth of the absorber, while the other half start at a depth of $22 \mathrm{~cm}$ from the front face of each detector. The segmentation of the HF calorimeters is $0.175 \times 0.175$, except for $|\eta|>4.7$, where it is $0.175 \times 0.35$. The HF energy resolution is $\sim 200 \% / \sqrt{E}$.

\section{Event selection and jet reconstruction}

The analysis [10] is performed using data collected in the total data sample collected at luminosities of about $10^{30} \mathrm{~cm}^{2} \mathrm{~s}^{-1}$ corresponds to an integrated luminosity of $(3.14 \pm 0.14) \mathrm{pb}^{-1}$. Events for the inclusive forward-jet analysis were selected with a trigger requiring a minimum jet transverse energy of $15 \mathrm{GeV}$ within $|\eta|<5.2$, while the events used in the dijet measurement were taken with a dijet trigger requiring two jets with summed calorimeter transverse energy above 
$30 \mathrm{GeV}$ also within $|\eta|<5$.2. The selected events were required to have a good primary vertex (PV) consistent with the measured transverse position of the beam: the PV was required to be reconstructed from at least 5 tracks and to lie within $24 \mathrm{~cm}$ in the longitudinal direction with respect to the nominal interaction point. This selection is highly efficient $(\approx 100 \%)$ for this analysis and rejects non-collision background. The events were required to contain at least ten tracks, of which at least $25 \%$ should satisfy the high purity requirement.

The central and forward jets were reconstructed with the anti- $k_{T}$ algorithm [11, 12] with a distance parameter $R=\sqrt{(\Delta \eta)+(\Delta \phi)}=0.5$. The algorithm was applied to calorimetric energy deposits. In the central region, jets are obtained from signals in calorimeter towers with energies in at least one HCAL cell, and from their geometrically corresponding ECAL crystals. In the forward region, jets are reconstructed using Cherenkov-light signals collected in both the HF short and long quartz readout fibres. All jets are required to have a transverse momentum $p_{T} \geq 35 \mathrm{GeV} / \mathrm{c}$.

\section{Results}

Following systematic effects are considered and the jet $p_{T}$ spectra which reconstructed from the calorimeter energies are corrected. The first one is, $p_{T}$ - and $\eta$-dependent response of the calorimeters, and possible overlap with other proton-proton interactions (pileup), and the second one is an "unfolding" of the impact of the jet energy resolution on the migration of events across $p_{T}$ bins. The reconstructed jet energy scale (JES) is first calibrated using data based on balancing the $p_{T}$ values in dijet and in photon-jet events, as well as from MC simulations [13]. The comparisons of the measured differential cross sections for jets before unfolding the energy resolution with the detector level MC simulations are shown in Fig. 1. The data shown are calibrated through the JES normalisation, but not unfolded. The cross sections in each interval of $\eta$ and $p_{T}$ are divided by their bin-widths. In the present analysis, the simulated events are normalised to the integrated luminosity. In order to account for the finite energy resolution of the calorimeters the second correction (unfolding) of the measured jet spectrum is applied. Although the bin size of the presented $p_{T}$ distributions equals or exceeds the experimental resolution, the combination of a steeply falling $p_{T}$-spectrum and calorimeter resolutions leads to migration of events across bins that can distort the distribution in $p_{T}$. Two methods are used to account for the bin-migration effect:

(i) Since the MC simulations (Fig. 1) reproduce the $p_{T}$-dependence of the inclusive forward-jet spectrum, and the simulated spectra for dijet events, the MC samples are used to study the bin-to-bin migrations.

(ii) The measured jet $p_{T}$ spectrum is fitted to some parameterised function $f\left(p_{T}\right)[15,16]$, the result of which can be smeared using the known (Gaussian) jet resolutions [17, 13]. The parameters of the model are then defined by fitting the smeared transverse $p_{T}$ spectrum $F\left(p_{T}\right)$ to the measured $f\left(p_{T}\right)$, and using the ratio of both distributions for the final correction [17].

The difference between the results of the two methods is below $10 \%$ for all $p_{T}$ bins. The factors obtained with the MC method are used to correct the mean values of $p_{T}$, while the results from the fits are used to assess the associated systematic uncertainties. The corresponding bin-bybin factors thus fully correct the jet spectrum from the detector to the particle levels via the factor 


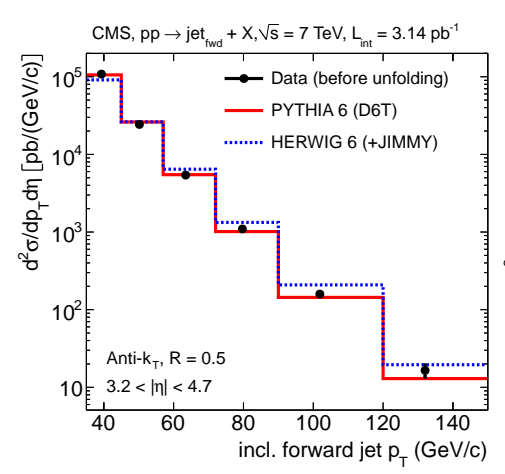

(a)

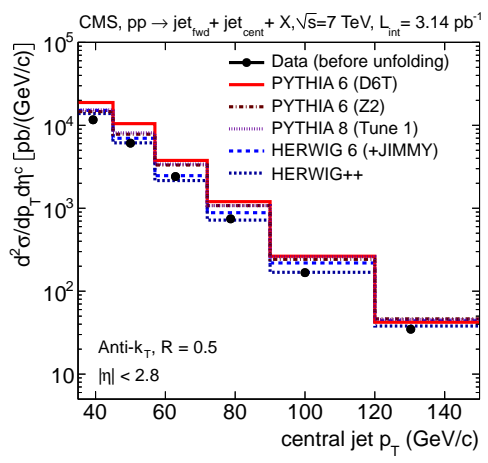

(b)

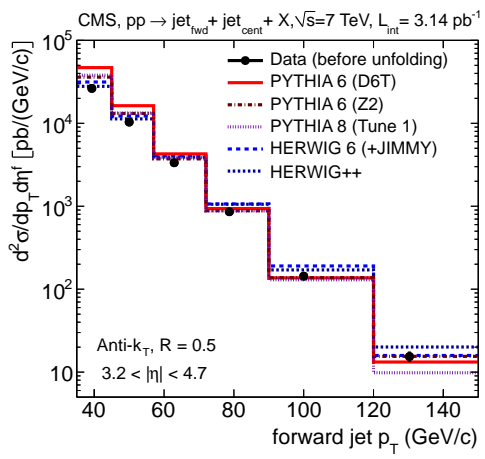

(c)

Figure 1: The reconstructed $p_{T}$ spectrum before unfolding for: (a) inclusive forward jets ,(b) central and (c) forward jets in dijet events.

$C_{\text {had }}\left(p_{T}, \eta\right)=\frac{N_{h d}^{\mathrm{MC}}\left(p_{T}, \eta\right)}{N_{\text {det }}^{\mathrm{MC}}\left(p_{T}, \eta\right)}$, where $N_{h a d}^{\mathrm{MC}}\left(p_{T}, \eta\right)$ and $N_{d e t}^{\mathrm{MC}}\left(p_{T}, \eta\right)$ are the jet event yields determined after hadronisation and after full simulation, respectively. The factor $N_{\text {had }}^{\mathrm{MC}}\left(p_{T}, \eta\right)$ is obtained by averaging the predictions from PYTHIA 6 with HERWIG 6+Jimmy, which provide different modelling of parton-to-hadron processes, one based on string and the other on cluster fragmentation, respectively.

The final differential inclusive jet cross section as a function of $\eta$ and $p_{T}$ :

$$
\frac{d^{2} \sigma}{d p_{T} d \eta}=\frac{C_{h a d}}{\mathscr{L} \cdot \varepsilon_{t}} \cdot \frac{N_{\text {evts }}}{\Delta p_{T} \cdot \Delta \eta}
$$

where $N_{\text {evts }}$ is the number of events. The factor $C_{\text {had }}$ accounts for bin-to-bin migrations due to $p_{T}$ resolution and detector to particle corrections. $\mathscr{L}$ is the total integrated luminosity, $\varepsilon_{t}$ is the efficiency of the jet trigger, and $\Delta p_{T}$ and $\Delta \eta$ are the sizes of the bins in $p_{T}$ and $\eta$, respectively. The jet triggers have a $\varepsilon_{t}=100 \%$ efficiency for all $p_{T}$ and $\eta$. Figure 2 shows the fully corrected and unfolded differential cross sections as a function of jet $p_{T}$ for inclusive jets at forward and central pseudorapidities. The ratio of theory to data for differential cross sections as a function of $p_{T}$, for inclusive jets at forward pseudorapidities (a), for central (b) and forward (c) jets produced in dijet events is shown in Fig. 3. Three primary sources of systematic uncertainty in the jet cross sections measurements are present:

- Uncertainty of JES: At forward rapidities, the HF calorimeter has a JES calibration uncertainty that varies between 3 and $6 \%$, depending on the $p_{T}$ and $\eta$ of the reconstructed jet [13]. The JES uncertainties of the central calorimeters have typical values between 2.5 and $3.5 \%$ [13]. The JES uncertainties, propagated to the steeply falling jet spectra (inverse power-law $p_{T}$ distributions with exponent of $n \approx 5$ ), translate into uncertainties of the order of $\pm(20-30) \%$ in the final forward and central jet cross sections.

- Uncertainty of unfolding and $p_{T}$ resolution: The $\pm 10 \%$ uncertainty on the jet $p_{T}$ resolution translates into an uncertainty of 3 to $6 \%$ (increasing with $p_{T}$ ) on the final cross sections. An additional uncertainty of $3 \%$, from the model dependence of the correction factors defined 
by the difference between the PYTHIA 6 and HERWIG 6 generators used to unfold the cross sections, is added in quadrature.

- Uncertainty of luminosity: The uncertainty of the integrated pp luminosity results in a $4 \%$ uncertainty on the overall normalisation of the spectra [14].

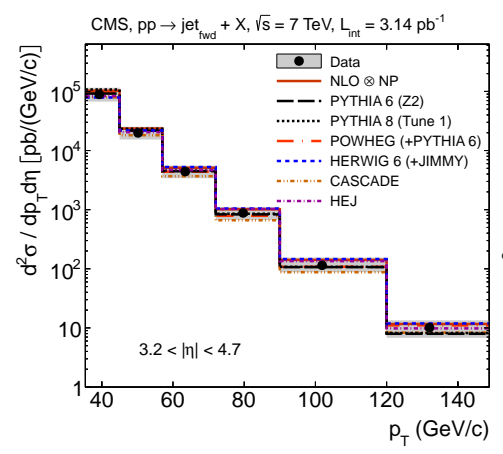

(a)

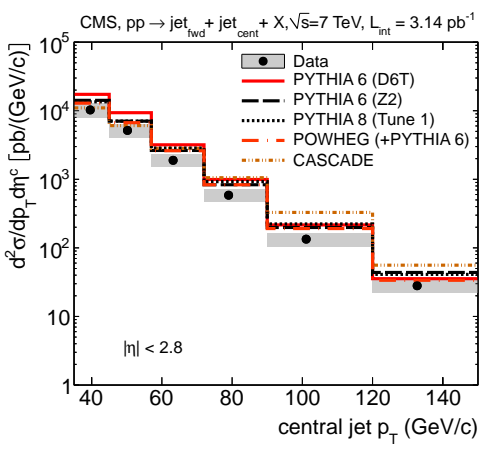

(b)

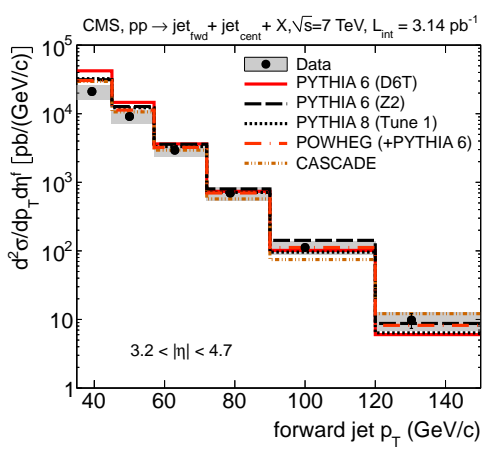

(c)

Figure 2: Fully corrected and unfolded differential cross sections as a function of jet $p_{T}$ for inclusive jets at forward pseudorapidities (a), for dijet events with at least one central jet (b) and one forward jet (c) compared to particle-level predictions. The error bars on all data points reflect just statistical uncertainties, with systematic uncertainties plotted as grey bands.

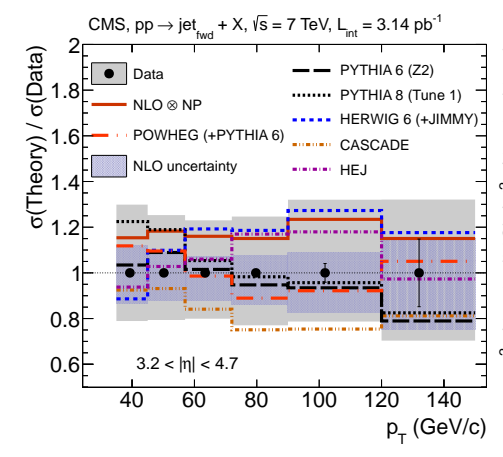

(a)

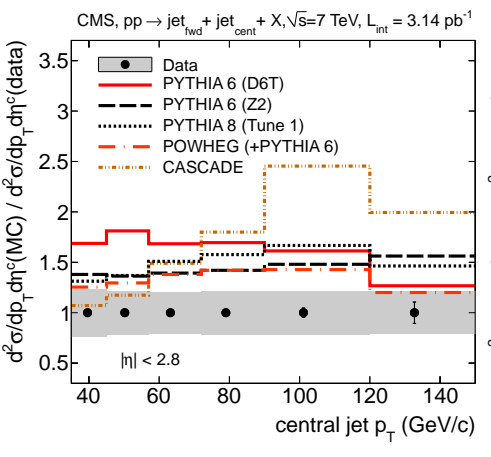

(b)

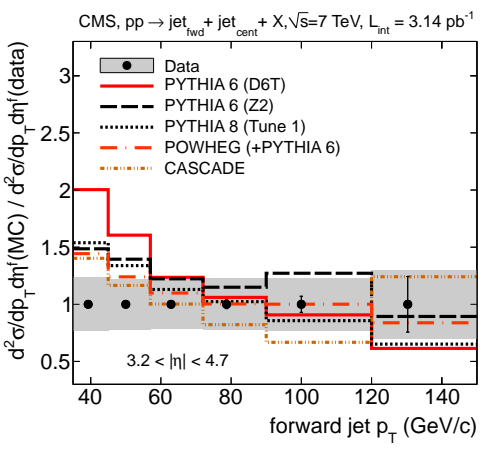

(c)

Figure 3: Ratio of theory to data for inclusive jets at forward pseudorapidities (a), for central (b) and forward (c) jets produced in dijet events.

\section{Conclusions}

Forward jet production in the $p_{T}$ range of $35-140 \mathrm{GeV} / \mathrm{c}$ has been measured by using $3.14 \mathrm{pb}^{-1}$ of data collected in proton-proton collisions at $\sqrt{s}=7 \mathrm{TeV}$. The total systematic uncertainties are of the order of $\pm 25 \%$ and dominated by the absolute jet energy scale. Within the current experimental and theoretical uncertainties, perturbative calculations reproduce globally well the measured forward jet cross section. The measurement provides a first test of perturbative QCD 
calculations in the forward region at the highest energies ever, as well as a first cross-check for QCD background estimates of other scattering processes, such as vector boson fusion, characterized by forward/backward jet production. The measurement of central forward jet associate cross section using $3.14 \mathrm{pb}^{-1}$ of data collected in pp collisions at $\sqrt{s}=7 \mathrm{TeV}$ is also presented. The total systematic uncertainty amounts to $30 \%$ dominated by the uncertainty of JES. The study provides cross section measurement for central and forward jets, compared to various Monte Carlo predictions, including Pythia 6 D6T tune, Pythia 6 Z2 tune, Pythia 8 tune1, Powheg+Pythia, Herwig++, Herwig+Jimmy, Powheg+Herwig, Cascade.

\section{References}

[1] D0 collaboration, V. Abazov et al., Phys. Rev. Lett. 101 (2008) 062001 [arXiv:0802.2400]. CDF collaboration, T. Aaltonen et al., Phys. Rev., D 78 (2008) 052006 [Erratum ibid. D 79 (2009) 119902], [arXiv:0807.2204].

[2] V. N. Gribov and L. N. Lipatov, Sov. Journ. Nucl. Phys. 15438 (1972); G. Altarelli and G. Parisi, Nucl. Phys. B126 298 (1977); Yu. L. Dokshitzer, Sov. Phys. JETP 46641 (1977).

[3] L. N. Lipatov, Sov. J. Nucl. Phys. 23338 (1976); E. A. Kuraev, L. N. Lipatov and V. S. Fadin, Zh. Eksp. Teor. Fiz 72 3(1977); Ya. Ya. Balitsky, L. N. Lipatov, Sov. J. Nucl. Phys. 28822 (1978).

[4] M. Ciafaloni, Nucl. Phys. B296 (1988) 49; S. Catani, F. Fiorani, and G. Marchesini, Phys. Lett. B234 (1990) 339; G. Marchesini, Nucl. Phys. B445 (1995) 49-80.

[5] F. Gelis, E. Iancu, J. Jalilian-Marian et al., Ann. Rev. Nucl. Part. Sci. 60 (2010) 463, [arXiv: 1002.0333].

[6] S. Cerci and D. d'Enterria, AIP Conf. Proc. 1105 (2009) 28, [arXiv:0812.2665].

[7] CMS collaboration, S. Chatrchyan et al. 2008 JINST 3 S08004.

[8] CMS collaboration, S. Chatrchyan et al., 2010 JINST 5 T03010 [arXiv:0910.3423]

CMS collaboration, S. Chatrchyan et al., 2010 JINST 5 T03012 [arXiv:0911.4991].

[9] G. Bayatian et al., Eur. Phys. J. C 53 (2008) 139.

[10] CMS collaboration, S. Chatrchyan et al., JHEP 06 (2012) 036 [arXiv:1202.0704].

[11] M. Cacciari, G.P. Salam and G. Soyez, JHEP 04 (2008) 063 [arXiv:0802.1189].

[12] M. Cacciari, G.P. Salam and G. Soyez, Eur. Phys. J. C 72 (2012) 1896 [arXiv:1111.6097].

[13] CMS collaboration, S. Chatrchyan et al., 2011 JINST 6 P11002 [arXiv:1107.4277].

[14] CMS collaboration, CMS-DP-2011-002, CERN, Geneva Switzerland (2011).

[15] S. Berman, J. Bjorken and J. B. Kogut, Phys. Rev. D 4 (1971), 3388.

[16] R. Feynman, R. Field, G. Fox, Phys. Rev. D 18 (1978) 3320.

[17] CMS collaboration, S. Chatrchyan et al., Phys. Rev. Lett. 107 (2011) 132001 [arXiv:1106.0208]. 